\title{
VEHICLE ROUTING IN PRACTICE: NEW SOLUTIONS AND CURRENT CHALLENGES
}

\author{
Achim Bachem, Universität zu Köln
}

In this talk we shall discuss two case studies of real life vehicle routing problems from different industry branches. We shall report on new solutions which solve online vehicle dispatch problems with time windows, truck dispatch problems in a network environment with many dispatchers and vehicle routing problems which are connected to a production planning system.

In addition we report on computational results of new parallel algorithms for solving complex vehicle routing problems, which we have implemented on a Parsytee 1024 processor system and on a workstation cluster using PVM.

The calculation of the distance resp. time matrix has to be done by using fast hierarchical shortest path algorithms on very large digitized street networks. We also have to take into account time depending travel times and traffic jams. We will present a model for simulating traffic which we have implemented on various supercomputer platforms.

Finally we shall present some of the needs for further research to develope computer aided dispatching systems in an industrial environment.

\section{References:}

1. A. Bachem, Hochstättler, and M. Malich, "The simulated trading heuristic for solving vehicle routing problems," Tech. Rep. 93-139, Mathematisches Institut, Universität zu Köln, Weyertal 8690, 50931 Köln, Germany, 1993.

2. K. Nagel, "Life-times of simulated traffic jams," to be published in: International Journal of Modern Physics C, no. 141, 1993.

3. K. Nagel, "Fast low fidelity microsimulation of vehicle traffic on supercomputers," Tech. Rep. 93140, Mathematisches Institut, Universität zu Köln, 1993.

4. G. Laporte, "The vehicle routing problem: An overview of exact and approximate algorithms," European Journal of Operational Research, vol. 59, pp. 345-358, 1992.

5. B. V. Cherkassky, A. V. Goldberg, and T. Radzik, "Shortest paths algorithms: Theory and experimental evaluation," tech. rep., Computer Science Department, Stanford University Stanford, CA 94305, USA, 1993. 